\section{Heart Failure}

\section{THE EFFECTS OF ATRIAL FIBRILLATION ON LEFT VENTRICULAR MECHANICS AND GENOMICS IN MITRAL REGURGITATION}

${ }^{1}$ Xuyu Jin, ${ }^{2}$ Jiangting Hu*, ${ }^{2}$ Francesco Pezzella, ${ }^{3}$ John Pepper. ${ }^{1}$ Oxford University, John Radcliffe Hospital; ${ }^{2}$ Oxford University; ${ }^{3}$ Royal Brompton Hospital

\subsection{6/heartjnl-2019-BCS.73}

Background The development of atrial fibrillation (AF) in mitral valve regurgitation (MR) adversely affects patient's long term outcome despite successful surgical repair. The fundamental changes in LV myocardial mechanics and genomics in $\mathrm{AF}$ remains to be fully elucidated. The aim of this study was to elucidate LV mechanics and myocardial gene expression profiling in MR patients with AF.

Material and methods We studied 28 consecutive patients (age $64 \pm 14$ yr, 18 males) undergoing elective MV surgery for degenerative severe MR. Of whom 25 patients (12 AF vs 13 SR) underwent physiological study using transoesophageal echo, high fidelity LV pressures and Swan-Ganz pulmonary catheter for cardiac output before the cardiopulmonary bypass. Transverse LV cavity dimension and wall thickness were derived from mid-cavity $\mathrm{M}$-mode echocardiograms along with LV pressure by digitising. Systolic wall stress, peak Vcf, thus myocardial power were determined. LV ejection fraction and MR fraction (MR volume/LVSV) were measured. Gene expression profiling by LV myocardium biopsy was studied in 11 patients using Stanford Human Exonic Evidence Based Oligonucleotide (HEEBO) array. Statistical analysis was performed between $5 \mathrm{AF}$ and $6 \mathrm{SR}$ cases using parametric permutative (permutation times=1000) t-test p-value cut-off 0.01. Gene functional annotation clusters were performed using Database for Annotation, Visualization and Integrated Discovery (DAVID, NIH, USA) and pathway analysed by GeneSpring GX10.

Results The AF group had a significantly greater MR fraction $(70 \pm 12$ vs $47 \pm 11, \%, \mathrm{p}<0.001)$, and greater mean myocardial power $(2.3 \pm 1.2$ vs $1.4 \pm 0.5$, watts, $\mathrm{p}=0.024)$, but had no difference in LVEF (65 \pm 8 vs $69 \pm 11, \%, \mathrm{p}=0.274)$ or systemic cardiac output $(3.4 \pm 0.8$ vs $3.8 \pm 0.8,1 / \mathrm{min}$, $\mathrm{p}=0.249$ ) when compared with those in SR group. The gene expression profiling demonstrated 149 genes deferentially expressed between AF and SR groups. Among which, 87 genes were up-regulated in AF and 62 genes were down regulated. Analysis by Direct Interaction network revealed several connected small island centred on genes like POLDIP2, UQCRFS1, RAMP1 and CALCR(up in AF) and TRAF5, MEF2A (down in AF). Further data mining using DAVID and the Significantly Enriched Core Pathways analysis showed that the up genes in AF were predominantly involved in the pathways of oxidative phosphorylation, mitochondrial respiratory chain complex, NADH dehydrogenase (ubiquinone) activity and metabolic pathways, whilst the down regulated genes in AF were presented in PI3K-Akt signalling pathway, focal adhesion and Glycoprotein/disulphide bond.

Conclusion When MR patients evolved from SR into AF, its MR severity increased significantly along with much greater LV mechanical energy output thus maintained systemic cardiac output. This is associated with significant up regulation of gene expression in mitochondrial oxidative phosphorylation, respiratory chain complex and $\mathrm{NADH}$ dehydrogenase, implying LV myocardium has become a major source of increased oxidative stress, whilst a lower expression of genes in PI3K-Akt signalling pathway also indicated a weakened crucial ROS-dependent oxidative stress regulatory mechanism for Redox. Taking together, we have demonstrated that $\mathrm{AF}$ in $\mathrm{MR}$ patients triggers a vicious cycle of myocardium energy output and ROS burden originated from the left ventricle. Our findings have added an important new dimension in the search for $\mathrm{AF}$ mechanism in MR.

Conflict of Interest none

\section{THE EFFICACY OF FRAILTY TOOLS IN DETECTING FRAILTY AND PREDICTING MORTALITY IN PATIENTS WITH CHRONIC HEART FAILURE}

${ }^{1}$ Shirley Sze*, ${ }^{2}$ Pierpaolo Pellicori, ${ }^{3}$ Jufen Zhang, ${ }^{4}$ Joan Weston, ${ }^{5}$ Andrew Clark. 'Leicester University Hospitals NHS Trust; ${ }^{2}$ Robertson Centre for Biostatistics; ${ }^{3}$ Anglia Ruskin University; ${ }^{4}$ Castle Hill Hospital; ${ }^{5}$ Department of Academic Cardiology, Hull York Medical School

\subsection{6/heartjnl-2019-BCS.74}

Introduction Frailty is common in patients with chronic heart failure (CHF) and is associated with adverse outcome. Many frailty tools are available, however, there is standard way of evaluating frailty in patients with CHF. Our study aims to report the prevalence of frailty, agreement and prognostic significance amongst 3 frailty assessment tools and 3 screening tools in CHF patients.

Methods We comprehensively studied frailty using 6 frailty tools. Frailty screening tools include: Clinical frailty scale (CFS); Derby frailty index \& Acute frailty network frailty criteria. Frailty assessment tools include: Fried criteria; Edmonton frailty score \& Deficit index. Since there is no gold standard in evaluating frailty in $\mathrm{CHF}$ patients, for each of the frailty tools, we used the results of the other 5 tools to produce a combined frailty index which we used as a "standard" frailty tool. Subjects were defined as frail if so identified by at least 3 out of 5 tools.

Results 467 consecutive ambulatory CHF patients (67\% male, median age 76 (IQR:69-82) years, median NTproBNP 1156 (IQR:469-2463) ng/L) and 87 controls (79\% male, median age 73 (IQR:69-77 years) were studied.

Prevalence of frailty was much higher in $\mathrm{CHF}$ patients than in controls $(30-52 \%$ vs $2-15 \%$, respectively). Amongst the frailty screening tools, DFI scored the greatest proportion of patients as frail (48\%) while CFS scored the lowest proportion as frail (44\%). Amongst the assessment tools, Fried criteria scored the greatest proportion of patients as frail (52\%) while EFS scored the lowest proportion as frail (30\%). (figure 1)

The prevalence of frailty was higher in patients with HeFNEF than HeFREF. The prevalence of frailty was higher in patients with atrial fibrillation (AF) than in those in sinus rhythm. The prevalence of frailty increased with 